\title{
Additional Chemotherapy with 5-FU plus Leucovorin between Preoperative Chemoradiotherapy and Surgery Improved Treatment Outcomes in Patients with Advanced Rectal Cancer
}

\author{
Song Ee Park ${ }^{*}$, Jin Hwa Choi ${ }^{2 *}$, Chang Hwan Choi ${ }^{1}$, Suk Won Park ${ }^{3}$, Beon Gyu Kim, Seong Jae Cha ${ }^{5}$, In \\ Gyu Hwang $1^{\bowtie}$ \\ 1. Department of Internal Medicine, Chung-Ang University College of Medicine, Seoul, South Korea \\ 2. Department of Radiation Oncology, Chung-Ang University Hospital, Seoul, South Korea \\ 3. Department of Radiation Oncology, Myongji Hospital, Gyeonggi-do, South Korea \\ 4. Department of Surgery, Chung-Ang University College of Medicine, Seoul, South Korea \\ 5. Department of Surgery, Hanyang university Hanmaeum Changwon Hospital, Changwon, South Korea \\ *Song Ee Park and Jin Hwa Choi Contributed equally to this work. \\ $\square$ Corresponding author: Division of Hemato-oncology, Department of Internal Medicine, Chung-Ang University College of Medicine, 224-1 Heukseok-dong, \\ Dongjak-gu, Seoul, South Korea. 06973. Tel: +82-2-6299-1403; Fax: 82-2-6299-2114; E-mail: oncology@cau.ac.kr \\ (c) Ivyspring International Publisher. This is an open access article distributed under the terms of the Creative Commons Attribution (CC BY-NC) license \\ (https://creativecommons.org/licenses/by-nc/4.0/). See http://ivyspring.com/terms for full terms and conditions.
}

Received: 2018.02.05; Accepted: 2018.10.01; Published: 2019.01.01

\begin{abstract}
Purpose: The aim of the preliminary study was to evaluate the efficacy and safety of 4-week chemotherapy with 5-Fluorouracil and leucovorin (LV5FU2) during the resting periods between preoperative CRT and surgery in patients with LARC.

Materials and Methods: Standard preoperative CRT was delivered to the entire pelvis at a total dose of 5040 Gy of radiation with concurrent 5-FU or capecitabine for 6 weeks. Twenty-three patients received additional preoperative chemotherapy with two cycles of 5-FU and LV (LV $200 \mathrm{mg} / \mathrm{m} 2$ and 5-FU bolus $400 \mathrm{mg} / \mathrm{m} 2$ on day 1 , and 5 -FU infusion $2400 \mathrm{mg} / \mathrm{m} 2$ for $46 \mathrm{hrs}$, every 2 weeks) after preoperative CRT. Surgery was performed at 2-4 weeks following the completion of preoperative chemotherapy.

Results: Between May 2013 and January 2015, 23 patients underwent preoperative CRT, with additional chemotherapy and surgery, and 23 patients completed the scheduled treatment. The median follow-up duration was 42.0 months. The tumor down-staging rate was observed in $65.2 \%$, and pathologic complete remission (PCR) was noted in 5 patients $(21.7 \%)$. T and $\mathrm{N}$ down-staging were observed in $16(69.6 \%)$ and $14(60.9 \%)$ patients, respectively. The four-year disease-free survival (DFS) rate was $73.9 \%$ and the four-year overall survival (OS) rate was $90.9 \%$ in patients who received additional chemotherapy. The four-year DFS rate was $100 \%$ in the tumor down-staging group vs. $25.0 \%$ in the non-down staging group treated with additional chemotherapy $(P<0.001)$. There was also a significant difference of the four-year OS rate $100 \%$ in the tumor down-staging group compared with $71.4 \%$ in the non-down staging group (P $=0.031$ ).

Conclusions: This preliminary study showed that additional preoperative chemotherapy with LV5FU2 was well tolerable and had an improvement in the downstaging rate and survival. Randomized controlled trial of this strategy is encouraged for definitive conclusions.
\end{abstract}

Key words: rectal cancer, preoperative therapy, chemotherapy, 5-fluorouracil (5-FU), leucovorin (LV)

\section{Introduction}

Preoperative chemoradiotherapy (CRT) has been used as a standard treatment in patients with locally advanced rectal cancer (LARC) because of increased local control, organ preservation and less toxicity [1]. 
In recent years, the tumor response to preoperative CRT has emerged as a potentially important predictive factor for local tumor control and survival. Strategies to achieve tumor down-staging may be clinically important. Patients showing tumor downstaging after preoperative CRT showed excellent survival benefits, and several studies demonstrated that tumor down-staging and pathologic complete response $(\mathrm{pCR})$ were significant prognostic factors for rectal cancer patients who received preoperative CRT $[2,3]$. The tumor down-staging rate has been reported as $65-85 \%$ for patients who received preoperative CRT [4-6]. An increased tumor down-staging rate may not only change definitive surgery strategies for anal preservation but also impact the survival rate [7]. Studies have demonstrated that the final pathologic stage remains the most significant prognostic factor in rectal cancer. Therefore, high tumor regression has been a significant goal in preoperative CRT for rectal cancer.

Conventional preoperative CRT has a resting period of 4-8 weeks after the completion of treatment [8]. The aim of the present study was to evaluate the efficacy and safety of additional 4-week chemotherapy with 5-Fluorouracil (5-FU) and leucovorin (LV) during the resting period between preoperative chemoradiotherapy (CRT) and surgery in patients with locally advanced rectal cancer (LARC). This approach would promote the potential antitumor regression effects of additional chemotherapy more than preoperative CRT alone. We propose that additional chemotherapy might increase tumor down-staging and represent a more effective sphincter-saving resection through a potential antitumor effect.

\section{Materials and Methods}

\section{Patients}

We performed a retrospective study in patients with a histologically proven, locally advanced adenocarcinoma of the rectum. Eligible patients had a histologically verified adenocarcinoma within $15 \mathrm{~cm}$ from the anal verge, with radiological evidence of a T3 or T4 tumor, or node positive status. Tumors were classified as lower $(<4 \mathrm{~cm}$ from the anal verge), middle (5-10 $\mathrm{cm}$ from the anal verge), and upper $(>10$ $\mathrm{cm}$ from the anal verge) rectal cancer according to their location. Other inclusion criteria were an Eastern Cooperative Oncology Group score (ECOG) of 0-1. Exclusion criteria included other coexisting malignancies or prior RT to the pelvis.

All patients underwent colonoscopy, carcinoembryonic antigen (CEA), rectal magnetic resonance imaging (MRI), abdomen computed tomography (CT) and chest CT scan at initial staging work up. In addition, all patients underwent KRAS mutation testing.

\section{CRT, additional chemotherapy and adjuvant chemotherapy}

Preoperative CRT was delivered to the entire pelvis at 45 Gy with daily doses of 1.8 Gy in 25 fractions, followed by a boost of 5.4 Gy in 3 fractions within 6 weeks, totaling 50.4 Gy with concurrent 5-FU or capecitabine for 6 weeks. Radiotherapy was administered 5 days per week from Monday to Friday with the weekend off. Patients received additional preoperative chemotherapy of two cycles of 5-FU and $\mathrm{LV}$ (LV $200 \mathrm{mg} / \mathrm{m}^{2}$ and $5-\mathrm{FU}$ bolus $400 \mathrm{mg} / \mathrm{m}^{2}$ on day 1, and 5-FU infusion $2400 \mathrm{mg} / \mathrm{m}^{2}$ for $46 \mathrm{hrs}$, every 2 weeks) after preoperative CRT. Surgery was performed at 2 4 weeks following the completion of additional chemotherapy. A lower anterior resection, a coloanal anastomosis or an abdominoperineal resection was performed on the principle of total mesorectal excision (TME). After surgery, the patients underwent adjuvant chemotherapy at four cycles of 5 -FU $500 \mathrm{mg} / \mathrm{m}^{2}$ every 4 weeks.

\section{Statistical analysis}

The primary endpoint of the present study was the tumor down-staging rate. The effect of preoperative CRT was assessed by using the pretreatment radiologic TNM stage and the postoperative pathologic TNM stage. We confirmed pathologic outcomes after additional chemotherapy to accurately assess the loco-regional nodal involvement of the rectum for down-staging. Down-staging was considered when pathologic $\mathrm{T}$ or $\mathrm{N}$ was lower than clinical $\mathrm{T}$ or $\mathrm{N}$. The secondary endpoints were the pathologic complete response ( $\mathrm{pCR}$ ) rate, disease-free survival (DFS), overall survival (OS), compliance and toxicity. DFS was estimated by considering the time from the start of preoperative CRT to relapse or death. OS was estimated by considering the time from the start of preoperative CRT to death. The rates of DFS and OS were calculated and compared by using the Kaplan-Meier methods and the log-rank test. The mDworak Tumor regression grade (TRG) system was graded as follows [9]: TRG 4, or complete regression, defined as no residual tumor cells in the primary tumor and regional LNs (ypTON0); TRG 3, or near complete regression, defined as one or two microscopic foci (each $<0.5 \mathrm{~cm}$ in diameter) of residual tumor cells or groups in the primary tumor and regional LNs; TRG 2, or moderate regression, defined as dominant fibroinflammatory changes with vasculopathy encompassing more than $50 \%$ of the entire tumor, including the tumor, regional $\mathrm{LN}$ 
metastases, and perirectal tumor deposits; and TRG 1, or minimal regression, defined as a dominant tumor mass encompassing more than $50 \%$ of the primary tumor and/or regional LN metastases. Toxicity was assessed according to the National Cancer Institute Common Toxicity Criteria, ver. 2.0 [10].

All statistical analyses were performed by using SPSS 24 (IBM Corp., Armonk, NY). DFS and OS were estimated by using Kaplan-Meier curves and a log-rank test. A priori sample size calculation was not possible as we had no reliable estimates for 4 weeks additional chemotherapy with 5-FU plus LV during the resting periods between preoperative CRT and surgery in patients with LARC. Thus, we performed a post hoc power estimate for tumor down-staging rate, OS and DFS. Post hoc power analysis indicated our study had over $90 \%$ power for tumor down-staging rate, OS and DFS (two-sided analysis and alpha value of 0.05). Power for tumor down staging rate was calculated based on the results of our study $(65.2 \%)$ and the historical data (30.0\%) [11]. And the power estimates for OS and DFS were based on the results of our study with accrual time 21 month and follow-up time 29 months. PASS $11^{\mathrm{TM}}$ software (NCSS, Kaysville, UT, USA) was used to perform the post-hoc power analysis. The study was approved by the Institutional Review Board of the Chung-Ang University College of Medicine. The requirement of informed consent was waived, as the study was based on retrospective analyses of existing administrative and clinical data.

\section{Results}

\section{Patients}

Between May 2013 and January 2015, 23 patients were enrolled and underwent surgery. The patient baseline characteristics are described in Table 1 . The median age was 59 years (range 38-81 years), and 15 $(62.2 \%)$ patients were male. All patients had good ECOG performance $0-1$. A total of $19(82.6 \%)$ patients had Clinical stage III, 4 (17.4\%) patients had stage II, and $8(34.8 \%)$ patients had CEA $>4 \mathrm{ng} / \mathrm{mL}$. Moreover, $17(73.9 \%)$ patients had KRAS wild type.

\section{Surgical findings}

All patients underwent surgery with TME. A sphincter-saving operative procedure in ultra-lower anterior resection and lower anterior resection was performed in $91.3 \%$ of patients (Table 2). A total of 8 patients had a tumor located $\leq 6 \mathrm{~cm}$ from the anal verge at the first staging. Two of the 8 patients in whom the tumor distal border was $\leq 3 \mathrm{~cm}$ from the anal verge had a sphincter-saving resection $(100 \%)$. An $\mathrm{R} 0$ resection was obtained in all cases, with negative distal and proximal margins.
Table 1. Baseline characteristics

\begin{tabular}{lll}
\hline Characteristics & & Patients $\mathbf{~ N = 2 3 )}$ \\
\hline Age & Redian age (year) & 59 \\
Gender, $\mathrm{n}(\%)$ & Female & $38-81$ \\
& Male & $8(34.8)$ \\
ECOG & 0 & $15(65.2)$ \\
& 1 & $9(39.1)$ \\
Tumor location & $\leq 4$ & $14(60.9)$ \\
from anal verge $(\mathrm{cm})$ & & $4(17.4)$ \\
& $5-10$ & \\
Clinical T stage & $\geq 11$ & $17(73.9)$ \\
& T2 & $2(8.7)$ \\
Clinical N stage & T3 & $2(8.7)$ \\
& T4 & $14(60.9)$ \\
& N0 & $7(30.4)$ \\
Clinical stage & N1 & $4(17.4)$ \\
& N2 & $11(47.8)$ \\
CEA & II & $8(34.8)$ \\
& III & $4(17.4)$ \\
KRAS & $\leq 4$ & $19(82.6)$ \\
& $>4$ & $15(65.2)$ \\
& Wild type & $8(34.8)$ \\
& Mutation & $17(73,9)$ \\
ECOG, Ean & Unknown & $3(13.0)$ \\
& & $3(13.0)$ \\
\hline
\end{tabular}

ECOG, Eastern Cooperative Oncology Group; CEA, Carcinoembryonic antigen; KRAS, Kirsten-ras.

Table 2. Surgical and pathologic characteristics

\begin{tabular}{lll}
\hline Characteristics & & Value, $\mathbf{n}(\%)$ \\
\hline Type of surgery & Ultra-lower anterior resection & $11(47.8)$ \\
& Lower anterior resection & $10(43.5)$ \\
& Abdominoperineal resection & $2(8.7)$ \\
Postoperative complication & None & $20(87.1)$ \\
& Anatomy site leakage & $1(4.3)$ \\
& Obstruction & $1(4.3)$ \\
Histologic type & Surgical site infection & $1(4.3)$ \\
& pCR & $5(21.7)$ \\
& Adenocarcinoma, WD & $3(13.0)$ \\
Pathologic T stage & Adenocarcinoma, MD & $14(61.0)$ \\
& Adenocarcinoma, PD & $1(4.3)$ \\
& pCR & $5(21.7)$ \\
Pathologic N stage & T0, Tis, T1 & $3(13.0)$ \\
& T2 & $5(21.7)$ \\
T3,4 & $10(43.5)$ \\
Stage & pCR & $5(21.7)$ \\
& N0 & $10(43.5)$ \\
N1 & $6(26.1)$ \\
Tumor regression grade & N2 & $2(8.7)$ \\
N down-staging & pCR & $5(21.7)$ \\
Down-staging & I & $8(34.8)$ \\
IIfferentiated; PD, poorly differentiated & $2(8.7)$ \\
& & $8(34.8)$ \\
& 2 & $1(4.3)$ \\
& 3 & $2(8.7)$ \\
& 4 & $7(30.4)$ \\
& & $7(30.4)$ \\
& & $6(26.1)$ \\
& & $16(69.6)$ \\
& & $14(60.9)$ \\
& & $15(65.2)$ \\
\hline
\end{tabular}

\section{Efficacy}

The pCR was noted in 5 patients $(21.7 \%)$, and 
down-staging was observed in 15 patients (65.2\%). $\mathrm{T}$ down-staging and $\mathrm{N}$ down-staging were observed in $16(69.6 \%)$ and $14(60.9 \%)$ patients, respectively (Table $2)$, and 6 (26.1\%) patients experienced relapse: 3 local and 3 distant liver recurrences. Patient correlations between clinical and pathological stages are described in Table 3. One patient was unable to complete the treatment regimen because of disease progression after 1 cycle of additional chemotherapy. The median follow-up duration was 42.0 months (range, 30.0-50.0 months) from the start of preoperative chemoradiotherapy. The four-year disease-free survival rate was $73.9 \%$ (Fig. 1). The four-year overall survival rate was $90.9 \%$, and 2 patients died. The median overall survival was not yet reached (Fig. 2). We performed univariate analyses to determine whether survival was affected by tumor down-staging in patients who received additional chemotherapy. The four-year DFS rate was $100 \%$ in the tumor down-staging group vs. $25.0 \%$ in the non-down-staging groups who received additional chemotherapy $(P<0.001)$. There was also a significant difference of the $100 \%$ four-year OS rate in the tumor down-staging group compared with the $71.4 \%$ rate observed in the non-down-staging group $(P=0.031)$.

\section{Compliance}

All patients completely received standard preoperative CRT and no dose reduction of chemotherapy or radiotherapy (Table 4). The median duration of preoperative chemoradiotherapy was 41 days (range 37 - 46 days), and 22 (95.6\%) patients completed additional chemotherapy with the scheduled treatment. One patient stopped additional chemotherapy due to progressed disease after 1 cycle of additional chemotherapy. Two patients received a $25 \%$ reduction in the starting dose due to nausea grade 3 and fatigue. All patients (100.0\%), including one patient with progressive disease, received surgery, and 22 patients $(95.6 \%)$ received adjuvant chemotherapy. The relative dose intensity of adjuvant chemotherapy was $82.5 \%$, and the median cycle was 4 (Table 4).

\section{Toxicity}

There was no case of additional chemotherapy delay due to chemotherapy toxicity. Postoperative complications were observed in 3 cases: one case of wound dehiscence, one case of postoperative bowel obstruction and one case of anastomotic leakage. Among patients administered adjuvant chemotherapy, 14 patients $(60.8 \%)$ received a dose reduction of adjuvant chemotherapy. Three patients (13.6\%) experienced anal bleeding during adjuvant chemotherapy, and these patients received a $25 \%$ dose reduction of chemotherapy in the next cycle. Two patients received a reduced chemotherapy dose due to weight loss. One patient developed Grade 3 mucositis (Table 5).
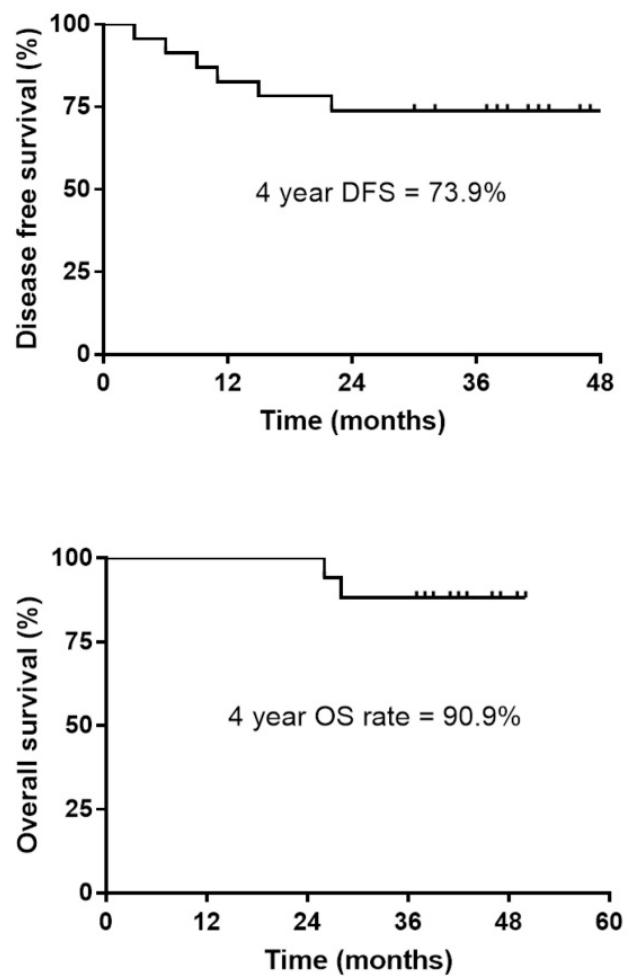

Figure 1. Disease-free survival (DFS) and Overall survival (OS) in patients who received additional chemotherapy.
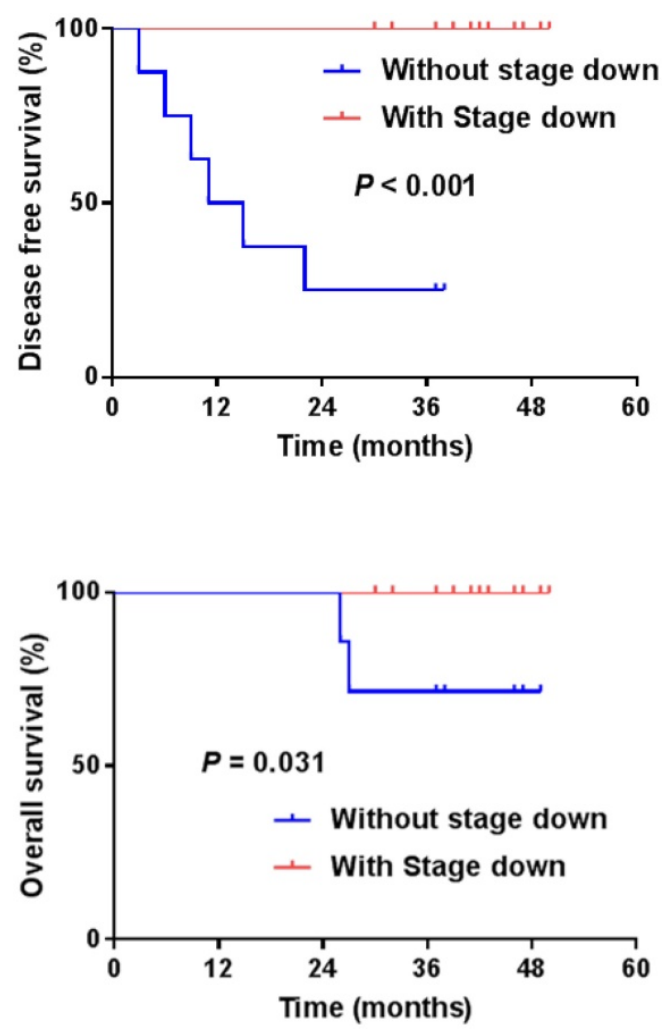

Figure 2. Disease-free survival (DFS) and Overall survival (OS) with or without tumor down-staging. 
Table 3. Correlation between clinical stage and pathologic stage

\begin{tabular}{|c|c|c|c|c|c|c|c|}
\hline \multirow{2}{*}{$\begin{array}{l}\text { Preoperative } \\
\text { clinical stages }\end{array}$} & \multicolumn{7}{|c|}{ Postoperative pathological stages } \\
\hline & T0 & Tis, T1 & T2 & T3, 4 & No & N1 & N2 \\
\hline $\mathrm{T} 2$ & 0 & 0 & 2 & 0 & & & \\
\hline T3 & 4 & 2 & 3 & 5 & & & \\
\hline $\mathrm{T} 4$ & 1 & 1 & 0 & 5 & & & \\
\hline No & & & & & 4 & 0 & 0 \\
\hline N1 & & & & & 8 & 3 & 0 \\
\hline N2 & & & & & 3 & 3 & 2 \\
\hline
\end{tabular}

Table 4. Compliance of CCRT, additional chemotherapy and adjuvant chemotherapy.

\begin{tabular}{|c|c|c|}
\hline & & $\begin{array}{l}\text { Patients } \\
(\mathrm{N}=23) \\
\text { Value, n (\%) }\end{array}$ \\
\hline \multirow[t]{3}{*}{ CCRT } & Received full dose of radiotherapy- no. (\%) & $23(100)$ \\
\hline & Received full dose of chemotherapy- no. (\%) & $23(100)$ \\
\hline & CCRT RDI & 100 \\
\hline \multirow[t]{14}{*}{$\begin{array}{l}\text { Additional } \\
\text { chemotherapy }\end{array}$} & $\begin{array}{l}\text { Patients who have completed planned } \\
\text { number of cycle }\end{array}$ & $22(95.6)$ \\
\hline & $\begin{array}{l}\text { Patients whose treatments were delayed at } \\
\text { any cycle }\end{array}$ & $0(0)$ \\
\hline & $\begin{array}{l}\text { Patients who had dose modification at any } \\
\text { cycle }\end{array}$ & $2(8.7)$ \\
\hline & Fluorouracil bolus & $2(8.7)$ \\
\hline & Fluorouracil continuous infusion & $2(8.7)$ \\
\hline & Total number of cycles given & 45 \\
\hline & Median number of cycles per patient (range) & $2(1-2)$ \\
\hline & Cycle with delayed schedule & 0 \\
\hline & Cycles with reduced doses & 2 \\
\hline & Fluorouracil bolus & $2(4.4)$ \\
\hline & Fluorouracil continuous infusion & $2(4.4)$ \\
\hline & Median dose $\left(\mathrm{mg} / \mathrm{m}^{2}\right.$ per day) [range] & \\
\hline & Fluorouracil bolus & $400[300-400]$ \\
\hline & Fluorouracil continuous infusion & $1200[900-1200]$ \\
\hline \multirow{3}{*}{$\begin{array}{l}\text { Adjuvant } \\
\text { chemotherapy }\end{array}$} & Received adjuvant chemotherapy & $22(95.6)$ \\
\hline & Adjuvant chemotherapy RDI & 85.2 \\
\hline & $\begin{array}{l}\text { Adjuvant chemotherapy median cycle, } \\
\text { (range) }\end{array}$ & $4(1-4)$ \\
\hline
\end{tabular}

Table 5. Acute toxicity during adjuvant chemotherapy $(n=22)$

\begin{tabular}{ll}
\hline Type of Toxic effect grade 3-4 & Value, $\mathbf{n}(\mathbf{4}) \mathbf{~ ( N = 2 2 )}$ \\
\hline Anal bleeding & $3(13.6)$ \\
Weight loss & $2(9.1)$ \\
ECOG performance increased & $2(9.1)$ \\
Mucositis & $1(4.5)$ \\
Dizziness & $1(4.5)$ \\
\hline
\end{tabular}

ECOG, Eastern Cooperative Oncology Group.

\section{Discussion}

We performed additional chemotherapy with 5-FU plus leucovorin for 2 cycles during the resting period for approximately 8 weeks after conventional preoperative CCRT until surgery for additional tumor size reduction and down-staging. In the present study, the pCR $(21.7 \%)$ was similar to that other studies; however, tumor down-staging (65.2\%), complete resection rate $(100 \%)$ and organ preservation $(91.3 \%)$ were improved compared to those in other studies $[1,12]$. Similar to the results of previous studies in which the survival rate was improved in patients exhibiting tumor down-staging [7], we showed a significant improvement in the four-year DFS and four-year OS rate in the tumor down-staging group.

Preoperative CRT is currently used as the standard treatment modality in patients with locally advanced rectal cancer; however, distant metastasis is reported in up to $30 \%$ of patients, and the survival benefit of preoperative CRT has not been demonstrated [1]. In the present study, the four-year disease free survival rate was $73.0 \%$, similar to the distant metastasis rate $(13.0 \%)$ reported in other studies [1].

We reported interesting results in terms of $\mathrm{pCR}$ rate $(21.7 \%)$ and down-staging (65.2\%). The downstaging rate after preoperative chemotherapy treatment was reported in 65 to $85 \%$ of cases [4, 5], with a pCR rate ranging from $20 \%$ in nonrandomized studies $[13,14]$ to 8 to $16 \%$ in randomized studies [15]. In other studies, capecitabine, an oral FU pro-drug, was administered at $825 \mathrm{mg} / \mathrm{m}^{2}$, delivered orally twice a day, as an additional chemotherapy during the resting period compared to a previous study on treatment with 5-FU and LV [12]. The pCR $(18.0 \%)$ and surgery rates $(80.0 \%)$ in a previous study with additional capecitabine treatment were comparatively low compared to the pCR rate $(21.7 \%)$ and surgery rate $(100.0 \%)$ in the present study.

Several studies with additional chemotherapy have been performed using FOLFOX [16], FOLFOX plus bevacizumab [17], and capecitabine [13]. In a previous study with FOLFOX plus bevacizumab for additional chemotherapy, pCR (25.0\%) was higher than the pCR $(21.7 \%)$ observed in the present study, but the morbidity $(21.4 \%)$ and mortality $(3.6 \%)$ rates were higher in the previous study than those in the present study. All patients enrolled in the present study were ECOG 0-1, which may affect data interpretation, as direct comparisons were difficult. However, the mortality was $0 \%$, showing a manageable toxicity of additional FL chemotherapy.

The incidence of sphincter preservation was $39 \%$ in preoperative CRT compared with $19 \%$ in postoperative CRT [1]. In the present study, a sphincter-saving procedure was highly performed $(91.3 \%)$ compared with an expected rate of $42 \%$ for pretreatment and an expected rate of $80 \%$ for additional chemotherapy with capecitabine $[1,12]$. In the present study, pCR was detected in 5 patients $(21.7 \%)$, and 4 of these patients $(80.0 \%)$ had baseline nodal involvement. We performed rectal MRI after additional chemotherapy to accurately assess the loco-regional nodal involvement of the rectum to clarify the down-staging results.

All patients received scheduled additional chemotherapy without delay or chemotherapy toxic- 
ity. There were no significant surgical complications after additional chemotherapy. Compared with other additional chemotherapy regimens [12], the incidence and severity of toxicity were low. After additional chemotherapy, the mortality until the end of adjuvant chemotherapy was $0 \%$ and no toxicity of grade 3 or higher was observed. All patients received surgery $(100 \%)$ with R0 resection, included one patient progressed disease with liver metastasis during additional chemotherapy.

This study has limitations that it was retrospective and single center data, meaning that it has lower statically power. Therefore these results should be interpreted with caution. In conclusion, the present study showed that additional preoperative chemotherapy with 5-FU and LV during the resting period after 6-week preoperative CRT was tolerable and active and favorably compares with conventional preoperative CRT. We showed a significant improvement in the four-year DFS and four-year OS rates in the tumor down-staging group. However, these results need to be confirmed in larger number, prospective, randomized controlled trials.

\section{Acknowledgement}

We gratefully acknowledege Hyun Kang for support with statistical analysis.

\section{Competing Interests}

The authors have declared that no competing interest exists.

\section{References}

1. Sauer R, Becker H, Hohenberger W, Rodel C, Wittekind C, Fietkau R, et al. Preoperative versus postoperative chemoradiotherapy for rectal cancer. The New England journal of medicine. 2004; 351: 1731-40.

2. Huebner M, Wolff BG, Smyrk TC, Aakre J, Larson DW. Partial pathologic response and nodal status as most significant prognostic factors for advanced rectal cancer treated with preoperative chemoradiotherapy. World journal of surgery. 2012; 36: 675-83.

3. Zorcolo L, Rosman AS, Restivo A, Pisano M, Nigri GR, Fancellu A, et al. Complete pathologic response after combined modality treatment for rectal cancer and long-term survival: a meta-analysis. Annals of surgical oncology. 2012; 19: 2822-32.

4. Kim JS, Kim JS, Cho MJ, Song KS, Yoon WH. Preoperative chemoradiation using oral capecitabine in locally advanced rectal cancer. International journal of radiation oncology, biology, physics. 2002; 54: 403-8.

5. Crane CH, Skibber JM, Birnbaum EH, Feig BW, Singh AK, Delclos ME, et al. The addition of continuous infusion 5-FU to preoperative radiation therapy ncreases tumor response, leading to increased sphincter preservation in locally advanced rectal cancer. International journal of radiation oncology, biology, physics. 2003; 57: 84-9.

6. Roh MS, Colangelo LH, O'Connell MJ, Yothers G, Deutsch M, Allegra CJ, et al. Preoperative multimodality therapy improves disease-free survival in patients with carcinoma of the rectum: NSABP R-03. Journal of clinical oncology: official journal of the American Society of Clinical Oncology. 2009; 27: 5124-30.

7. Rodel C, Martus P, Papadoupolos T, Fuzesi L, Klimpfinger M, Fietkau R, et al. Prognostic significance of tumor regression after preoperative chemoradiotherapy for rectal cancer. Journal of clinical oncology: official journal of the American Society of Clinical Oncology. 2005; 23: 8688-96.

8. Glimelius B. Optimal Time Intervals between Pre-Operative Radiotherapy or Chemoradiotherapy and Surgery in Rectal Cancer? Frontiers in oncology. 2014; 4: 50.

9. Kim SH, Chang HJ, Kim DY, Park JW, Baek JY, Kim SY, et al. What Is the Ideal Tumor Regression Grading System in Rectal Cancer Patients after Preoperative Chemoradiotherapy? Cancer research and treatment: official journal of Korean Cancer Association. 2016; 48: 998-1009.
10. Trotti A, Byhardt R, Stetz J, Gwede C, Corn B, Fu K, et al. Common toxicity criteria: version 2.0. an improved reference for grading the acute effects of cancer treatment: impact on radiotherapy. International journal of radiation oncology, biology, physics. 2000; 47: 13-47.

11. Theodoropoulos G, Wise WE, Padmanabhan A, Kerner BA, Taylor CW, Aguilar PS, et al. T-level downstaging and complete pathologic response after preoperative chemoradiation for advanced rectal cancer result in decreased recurrence and improved disease-free survival. Diseases of the colon and rectum. 2002; 45: 895-903.

12. Zampino MG, Magni E, Leonardi MC, Petazzi E, Santoro L, Luca F, et al. Capecitabine initially concomitant to radiotherapy then perioperatively administered in locally advanced rectal cancer. International journal of radiation oncology, biology, physics. 2009; 75: 421-7.

13. De Paoli A, Chiara S, Luppi G, Friso ML, Beretta GD, Del Prete S, et al. Capecitabine in combination with preoperative radiation therapy in locally advanced, resectable, rectal cancer: a multicentric phase II study. Annals of oncology: official journal of the European Society for Medical Oncology. 2006; 17: 246-51.

14. Carraro S, Roca EL, Cartelli C, Rafailovici L, Castillo Odena S, Wasserman E, et al. Radiochemotherapy with short daily infusion of low-dose oxaliplatin, leucovorin, and 5-FU in T3-T4 unresectable rectal cancer: a phase II IATTGI study. International journal of radiation oncology, biology, physics. 2002; 54: 397-402.

15. Gerard JP, Conroy T, Bonnetain F, Bouche O, Chapet O, Closon-Dejardin MT, et al. Preoperative radiotherapy with or without concurrent fluorouracil and leucovorin in T3-4 rectal cancers: results of FFCD 9203. Journal of clinical oncology: official journal of the American Society of Clinical Oncology. 2006; 24: $4620-5$.

16. Cancer, Leukemia Group B, Ryan DP, Niedzwiecki D, Hollis D, Mediema BE, et al. Phase I/II study of preoperative oxaliplatin, fluorouracil, and external-beam radiation therapy in patients with locally advanced rectal cancer: Cancer and Leukemia Group B 89901. Journal of clinical oncology : official journal of the American Society of Clinical Oncology. 2006; 24: 2557-62.

17. Liang JT, Lai HS, Cheng KW. Technical feasibility of laparoscopic total mesorectal excision for patients with low rectal cancer after concurrent radiation and chemotherapy with bevacizumab plus FOLFOX. Surgical endoscopy. 2011; 25: 305-8. 